\title{
La lógica difusa aplicada a los ratios financieros en el sector cooperativo del Ecuador
}

\section{Fuzzy logic applied to financial ratios in the cooperative sector of Ecuador}

\author{
Jaime Díaz Córdova \\ Edisson Coba Molina \\ Klever Moreno Gavilanes \\ Edwin Santamaría Freire \\ Universidad Técnica de Ambato, Ecuador \\ Autor por Correspondencia: jaimefdiaz@uta.edu.ec, edisoncoba@uta.edu.ec, \\ kleveramoreno@uta.edu.ec, edwinjsantamaria@uta.edu.ec \\ Fecha de recepción: 27 de Marzo de 2017 - Fecha de aceptación: 5 de Junio de 2017
}

Resumen: Al hablar de lógica difusa se debe deducir que está herramienta propone un horizonte más amplio que la lógica normal, permitiendo la utilización de la lógica cotidiana, dentro. Esta metodología busca una forma distinguida de obtener una respuesta a partir de información imprecisa o ambigua. El estudio tiene por objeto evaluar los ratios del segmento 1 de las Cooperativas de Ahorro y Crédito del Ecuador en un rango de bajo, estable y óptimo, mediante grados de pertenencia y variables lingüísticas que sustentan la conversión de los índices financieros a términos difusos. Para la elaboración del estudio se tomaron en consideración los principales ratios del sector cooperativo, basados en las metas del sistema CAMEL, los cuales a través de la función triangular de la lógica difusa obtienen grados de pertenencia donde se sitúan los ratios del segmento. Los resultados obtenidos en cuanto al índice de liquidez es $<$ bajo $>$ lo que podría significar poca disponibilidad de fondos. Por otra parte, los ratios de eficiencia financiera y estructura y calidad de activos se ubicaron en un nivel <estándar> lo que representaría solidez en ambos ratios. En tanto los índices de rentabilidad, cobertura de provisiones para cartera improductiva rentabilidad y eficiencia microeconómica alcanzaron los resultados esperados estableciéndose en un nivel <óptimo> lo que significaría que estos ratios se encuentran saludables.

Palabras Clave: lógica difusa; ratios financieros; variables linguiísticas

Abstract: When we refer to fuzzy logic we must conclude that this tool offers a wider than normal logic horizon, allowing the use of everyday logic. This methodology seeks a distinguished way to get an answer from inaccurate or ambiguous information. The study aims to assess the ratios of segment 1 of the credit union of Ecuador in a range of low, stable and optimal, by degrees of membership and linguistic variables that support the conversion of financial indices vague terms. For the preparation of the study were considered key ratios of the cooperative sector, based on the goals of the CAMEL system, which through the triangular function of fuzzy logic membership degrees obtained where the ratios of the segment are located. The results obtained in terms of liquidity ratio are low which could mean limited availability of funds. Moreover financial efficiency ratios and asset quality structure and stood at a standard level which would represent strength in both ratios. While profitability ratios, coverage provisions for unproductive portfolio profitability and microeconomic efficiency achieved expected results established at an optimum level which would mean that these ratios are healthy

Key words: fuzzy logic; financial ratios; linguistic variables 


\section{Introducción}

Al hablar de lógica difusa se inicia explicando las variables denominadas lingüísticas que permiten analizar perspectivas numéricas de cero (0) a uno (1) con intervalos de medición (Trillas \& Gutierrez , 1992). Estas variables lingüísticas permiten al experto establecer una valoración cualitativa de datos numéricos que pueden medir el comportamiento difuso de cualquier área de conocimiento como el Control Industrial área donde este tipo de lógica tiene varios seguidores (Duarte, 2000). En México, por ejemplo, la lógica difusa se utilizó para ingeniería como método de toma de decisiones (Guzman \& Castaño, 2006). Otra aplicación de la lógica borrosa es como alternativa para modelar la información imprecisa (Aceves, 2001). Es por esta razón que el presente estudio aplica la lógica difusa en el área financiera.

Al analizar las razones financieras de una entidad, estos valores identifican las condiciones empresariales en forma de cumplimiento de metas con base a modelos de medición como el CAMEL (Richardson, 2009). Sin embargo, al realizar el análisis financiero tradicional a través de ratios puede omitirse información relevante al momento de tomar una decisión. En este sentido la lógica difusa puede constituirse en una herramienta que permita dar una mejor perspectiva en cuanto a la toma decisiones (Pérez \& León, 2007), para realizar un análisis más profundo de la situación financiera de una organización. De ello, se plantea por objeto de estudio, evaluar los índices financieros del segmento 1 de las Cooperativas de Ahorro y Crédito del Ecuador utilizando lógica difusa que permite dar una categoría y una mejor interpretación al indicador para medir su nivel.

\section{Lógica Difusa}

Este tipo de lógica aparece por primera vez a mediados de los años sesenta gracias a la investigación de Lotfy A. Zadeh, quien en un inicio la llamó incompatibilidad. Tiempo después introdujo el concepto difuso, en su publicación "Fuzzy Sets" en 1965. A partir de su aparición innumerables investigadores se dedicaron a explorar nuevas teorías referidas a este tipo de lógica (Benito Matías \& Duran Vicente) ya que los elementos en los que se basa no son números sino etiquetas lingüísticas. Esta idea es la que permite que el conocimiento pueda presentarse de forma cualitativa en un lenguaje matemático (Hurtado, Quintero, \& Garcia , 2014) y aunque son más imprecisos que los números, muchas veces son más fáciles de entender para el razonamiento humano. (Morales, 2002)

La lógica borrosa o difusa es una alternativa a la lógica clásica. La base de la lógica difusa son los conjuntos difusos, cuya utilidad es trabajar con información que difiere a la lógica tradicional. En el mundo en que vivimos existe mucho conocimiento ambiguo e impreciso, el mismo razonamiento humano que actúa con este tipo de información es por ello que la lógica difusa fue diseñada para imitar este comportamiento (Reyes Vera, 1994). La lógica difusa contiene al lenguaje ordinario y comprensible de la cotidianeidad, con la determinación de cuan verdadera o falsa es la información emitida, donde también puede aplicarse al estudio y análisis económico de una entidad financiera. Motivo por el cual no es fácil en la lógica normal explicar ciertos tipos de conceptos adaptados a este caso (Haank, 1996)

La metodología difusa es usada comúnmente en casos cuya complejidad en el proceso de análisis es muy alta y no se puede precisar con modelos matemáticos tradicionales, dando paso 
de esta forma a conceptos subjetivos para la precisión del análisis (D`Negri \& De Vito, 2006). Donde, se puede establecer variables que ayudan a la comprensión total y simplificada de un hecho, pudiendo ser posible o no ser posible con escalas de 0 a 1 donde el cero significa "no pertenencia" y uno es la "total pertenencia". Lo cual hace que números intermedios como 0,5 entren en un estado de incertidumbre o ambigüedad adquiriendo diferentes significancias (Jiménez, Vallejo, Albusac, Castro-Schez, \& Glez-Morcillo, 2011). A estas variables se les conoce como variables de incidencia. (Lopez, 2013)

\section{Variables de Incidencia}

Las variables de incidencia llamadas también variables difusas utilizan un concepto análogo, son útiles en momentos de precisión donde una palabra no satisface" (Trillas \& Gutierrez, 1992). Las variables de incidencia dan paso a los grados de incidencia que van de cero (0) a uno (1) (Kaufmann \& Gil-Aluja, 1988).

Bajo esta consideración aparecen las variables lingüísticas que se caracterizan por dar valores cualitativos a lo cuantitativo a través de etiquetas lingüísticas que evalúen el nivel de desempeño de un fenómeno (Galindo, 2002). El objetivo de una variable de incidencia es ayudarse en los modificadores lingüísticos para evitar la ambigüedad de conceptos (Lopez , 2013). El significado de cada etiqueta lingüística se determina a través de conjuntos difusos para lo cual se debe de constituir medidas de incertidumbre que sean más ajustadas a la realidad, para obtener estos niveles más precisos se usan modificadores lingüísticos como <muy>, <más o menos>, <bastante>, <extremadamente>, entre otros. (Mullor, 2000)

Una variable lingüística cuyos posibles valores son palabras, está ligada estrechamente a las funciones de pertenencia que son una forma de representar de forma gráfica un conjunto borroso (Blass, 1993)

\section{Funciones de Pertenencia}

Hay funciones típicas o propicias de los conjuntos borrosos ya sea por la factibilidad de su uso o la complementación con respecto a la estructura de esta lógica, son capaces de definir el valor linguístico asociado al conjunto. Existen varios modelos para representar de forma lineal los conjuntos borrosos, entre estos la representación triangular y la trapezoidal (Cerra, Orizaola, \& Jimenez, 2014).

Con base en la pendiente de cada recta es posible definir el grado de pertenecía de un conjunto borroso como es el caso del modelo triangular que para su determinación utiliza las funciones de pertenencia; Gamma, L Y Triangular. Dentro del modelo triangular se definen intervalos, conocidos como grados de pertenencia (Gonzales, 2012). Los grados de pertenencia están acompañados de una variable lingüística (Blass, 1993)

La Función Gamma es una extensión de la función factorial de números complejos y reales (Marzan, 2011). Función L= 1- la función Gamma: determina el valor de una pendiente de valores con tendencia a cero. Función Triangular: representa velocidades de subida y bajada constantes (Garcia, Trinidad, \& Gomez, 2004). 
Los Grados de pertenencia son conjuntos que se caracterizan por una función de pertenencia, es decir que asigna a cada objeto un grado variante entre cero y uno (Pérez \& León, 2007).

\section{Análisis de la Información Financiera}

Un análisis financiero permite evaluar la posición financiera y los resultados de una entidad en base a sus operaciones, cuyo objetivo es detectar las fortalezas y debilidades de la misma (Villegas, 2002). Este tipo de análisis se fundamenta en los Estados Financieros, con base

al cálculo de ratios financieros que interpretados expresan la situación económica de una entidad (Nava \& Marbelis, 2009) .

Con base en lo mencionado por Alarcón \& Ulloa (2012) manifiestan que este tipo de análisis, estudia la rentabilidad y la liquidez de una entidad, además visualiza como la administración utiliza con eficiencia sus recursos económicos, ayudando a formular estrategias futuras mediante el empleo de métodos como el análisis vertical, análisis horizontal y ratios financieros entre otros.

La relación que existe entre dos números forma un ratio o razón que es el resultado de relacionar dos cuentas de los estados de una entidad. Los ratios o índices financieros son capaces de proveer información que permita tomar decisiones de forma acertada a quienes la administran (Aching, 2006). La utilidad de los índices financieros es mejorar el desempeño de las organizaciones, requiere una base de datos de la información financiera para su operación (Bernal \& Amat, 2012). Constituyen el eje fundamental para la toma de decisiones (Santandreu, 2000). Los ratios se calculan en base a fórmulas de tal manera que existen ratios para el sector no financiero y financiero.

\section{Modelos de Análisis de la Estructura Financiera}

A partir de la utilización de los ratios como mecanismo para evaluar la situación financiera de una entidad cooperativa nacen métodos que permiten medir varias áreas de una entidad de Ahorro y Crédito, entre los de mejor aplicación se encuentran el Método PERLAS y CAMEL.

El método CAMEL tiene la ventaja de resumir en un solo indicador la situación general de una entidad financiera, fue introducido en la década de los 80 por los tres organismos de supervisión y regulación de los Estados Unidos, el Sistema de Reserva Federal (FED), la Oficina de Control de la Moneda (OCC) y la Corporación Federal de Seguro de Depósito (FIDC) (Unidad de Gestión del Riesgo del Sistema Financiero, 2015).

El método CAMEL proporciona un marco metodológico que consiste en evaluar cinco aspectos claves de la calidad financiera de una entidad bancaria, como son: Ajuste de Capital, Calidad de los Activos, Gestión Administrativa, Ganancias y Liquidez, respectivamente (Buniak). La metodología CAMEL se encuentra presente en países como Venezuela, México, Ecuador, entre otros Los ratios más representativos dentro del análisis CAMEL utilizados para el 
estudio se presentan en la Tabla 1 con sus respectivas metas y fórmulas de cálculo de cada indicador

Tabla 1 Metas de los Indicadores Financieros método CAMEL

\begin{tabular}{|c|c|c|c|}
\hline \multicolumn{4}{|c|}{ Sector Financiero } \\
\hline \multicolumn{2}{|c|}{ Nombre Del Indicador } & \multirow{2}{*}{$\begin{array}{l}\text { Fórmula } \\
\text { Activos productivos / pasivos con costo }\end{array}$} & \multirow{2}{*}{$\begin{array}{c}\text { Metas } \\
\text { Camel } \\
208,79 \%\end{array}$} \\
\hline Estructura y calidad de activos & Activo Promedio $=$ & & \\
\hline $\begin{array}{l}\text { Cobertura de provisiones para } \\
\text { cartera improductiva }\end{array}$ & $\begin{array}{l}\text { Cartera Comercial } \\
=\end{array}$ & Cobertura de la cartera problemática & $29,04 \%$ \\
\hline Eficiencia Microeconómica & $\begin{array}{l}\text { Gastos de } \\
\text { Operación = }\end{array}$ & Gastos de operación / Margen financiero & $96,39 \%$ \\
\hline Rentabilidad & $\begin{array}{l}\text { Patrimonio } \\
\text { Promedio = }\end{array}$ & $\begin{array}{l}\text { Resultado del ejercicio / Patrimonio } \\
\text { promedio }\end{array}$ & $0,74 \%$ \\
\hline Liquidez & $\begin{array}{l}\text { Fondos disponibles } \\
=\end{array}$ & $\begin{array}{l}\text { Fondos Disponibles / Total depósitos a } \\
\text { corto plazo }\end{array}$ & $44,41 \%$ \\
\hline Eficiencia financiera & $\begin{array}{l}\text { Suficiencia } \\
\text { Patrimonial }=\end{array}$ & $\begin{array}{l}\text { Margen de intermediación estimado / } \\
\text { Patrimonio promedio }\end{array}$ & $620,75 \%$ \\
\hline
\end{tabular}

Fuente: Elaboración propia a partir de Andrade (2014)

\section{Sector Financiero Popular y Solidario en el Ecuador}

Las Finanzas Populares y Solidarias constituyen un mecanismo de organización y participación de los actores y sujetos sociales de la Economía Popular en el Sistema Financiero (PNFPEES, SENAMI, \& PNUD, 2012). En Ecuador, son cooperativas las organizaciones económicas solidarias constituidas con finalidad social y sin fin de lucro, auto gestionadas democráticamente por sus socios que buscan el beneficio de sus integrantes (LOEPS, 2011). Miño (2013) menciona que, el origen del cooperativismo en Ecuador, se remonta a la organización comunitaria indígena que utilizó formas de cooperación en la construcción de caminos, viviendas, acequia y múltiples obras sociales de larga duración.

Para el año 1972 en Ecuador ya estaban constituidas las más grandes cooperativas. En 1999 se constituye la Asociación Nacional de Cooperativas de Ahorro y Crédito (ASOCOAC) que agrupaba a las cooperativas supervisadas por la Superintendencia de Bancos y Seguros, durante el mismo año se crea la Federación de Cooperativas de Ahorro y Crédito (FECOAC) (La Hora, 2006) organismo impulso la creación del sistema cooperativo, conformado por corporaciones de servicios especializados a fin de atender con asistencia técnica a las cooperativas de ahorro y crédito, el objetivo no se alcanzó debido a la débil coordinación organizacional (Miño Grijalva, 2013).

En el año 2006, el establecimiento de un nuevo marco constitucional logrado por el Movimiento Alianza País determina una visión social lo que dio paso a la expedición de la Ley 
Orgánica de la Economía Popular y Solidaria y del Sector Financiero Popular y Solidario que se maneja hasta la actualidad (Miño Grijalva, 2013). El nacimiento del sistema de crédito cooperativo en el Ecuador es una respuesta social para suplir la falta de fuentes de liquidez, para evitar los abusos de prestamistas usureros, en el transcurso de su desarrollo histórico el sector cooperativo experimento trasformaciones (LOEPS, 2011).

\section{Metodología}

\section{Cooperativas dentro del Estudio}

En el Ecuador 917 organizaciones corresponden al sector financiero. Las COAC conforman un segmento en crecimiento que representa el $28 \%$ del Sector Cooperativo y Solidario SEPS (Revista Lideres, 2015).

El sector cooperativo en Ecuador se encuentra dividido en cinco segmentos con relación al activo (SEPS, 2015). Los segmentos son determinados por la Junta de Política y Regulación Monetaria Financiera la misma que en febrero de 2015 determino que el segmento uno estaría conformado por las entidades con activos superiores a USD 80`000.000,00, este monto está sujeto a actualización anual.

Tabla 2 Segmentos del Sector Cooperativo

\begin{tabular}{|c|c|}
\hline Segmento & Activos (USD) \\
\hline 1 & Mayor a $80^{\prime} 000.000,00$ \\
\hline 2 & Mayor a $20^{\prime} 000.000,00$ hasta $80^{\prime} 000.000,00$ \\
\hline 3 & Mayor a $5^{\prime} 000.000,00$ hasta $20^{\prime} 000.000,00$ \\
\hline 4 & Mayor a $1^{\prime} 000.000,00$ hasta $5^{\prime} 000.000,00$ \\
\hline 5 & Hasta $1^{\prime} 000.000,00$ \\
\hline
\end{tabular}

Fuente: SEPS (2015)

Para el presente estudio se tomó como muestra el segmento 1 de las cooperativas de ahorro y crédito dado que es el segmento con mayor representatividad de la economía de Ecuador. Este segmento que se encuentra bajo la supervisión de la economía popular y solidaria (SEPS) está integrado por 24 cooperativas de ahorro y crédito.

Tabla 3 Cooperativas del segmento 1

\begin{tabular}{ll}
\hline Juventud Ecuatoriana Progresista Ltda. & 23 De Julio Ltda. \\
Jardín Azuayo Ltda. & Andalucía Ltda. \\
San José Ltda. & Cooprogreso Ltda. \\
De La Pequeña Empresa Biblián Ltda. & Alianza Del Valle Ltda. \\
Pablo Muñoz Vega Ltda. & 29 De Octubre Ltda. \\
\hline
\end{tabular}




\begin{tabular}{ll}
\hline Tulcán Ltda. & Policía Nacional Ltda. \\
De La Pequeña Empresa De Cotopaxi Ltda. & De Los Servidores Públ. Del Minist. De Educ. y Cultura \\
Riobamba Ltda. & Oscus Ltda. \\
Santa Rosa Ltda. & San Francisco Ltda. \\
Atuntaqui Ltda. & El Sagrario Ltda. \\
Pilahuin Tío Ltda. & Cámara De Comercio De Ambato Ltda. \\
Vicentina Manuel Esteban Godoy Ortega Ltda. & Mushuc Runa Ltda. \\
\hline
\end{tabular}

Fuente: Elaborado a partir de la SEPS (2015)

El valor de cada ratio financiero será tomado de las metas establecidas según el Método CAMEL. Las metas serán consideradas como un punto medio o estándar. Los indicadores sujetos de análisis, se consideraron según su relevancia uno por cada grupo es decir de Liquidez se analizó Fondos Disponibles, en cuanto a Eficiencia Financiera se tomó Suficiencia Patrimonial, para rendimiento de cartera se dio prioridad a Cartera de Comercial por Vencer. Los valores de cada ratio reflejaron a través de las variables de incidencia el nivel en que se encuentra el indicador. Las variables de incidencia significan cualquier valor que esté basado en la percepción humana (Lopez , 2013). Las variables de incidencia o difusas utilizadas para la investigación poseen rangos de: cero a cero coma tres (0-0,3) un valor lingüístico de "bajo", de cero coma cuatro a cero coma seis $(0,4-0,6)$ una significancia de "estable" y los valores de cero coma siete a uno (0,7-1), "optimo". En la tabla 4 se encuentran detallados los valores de pertenencia de las variables de incidencia.

Tabla 4 Variables de incidencia y Grados de Pertenencia

\begin{tabular}{lcc}
\hline Conceptualización & $\begin{array}{c}\text { Grados de } \\
\text { Pertenencia }\end{array}$ & $\begin{array}{c}\text { V. } \\
\text { Lingüística }\end{array}$ \\
\hline $\begin{array}{l}\text { Mecanismo que sirve para encontrar un sistema de la toma de decisiones } \\
\text { (Alarcón \& Ulloa, 2012) que identifica incidencias directas de causa y efecto } \\
\text { (Aching, 2012) }\end{array}$ & $0-0,3$ & Bajo \\
& $0,4-0,6$ & Estable \\
& $0,7-1$ Optimo
\end{tabular}

Fuente: Autores

Con el objeto de simplificar el tratamiento de los índices, serán resumidos en siglas.

Tabla 5 Descripción de las siglas

\begin{tabular}{llll}
\hline Siglas & Descripción & Siglas & Descripción \\
\hline LZ & Liquidez & AP & Activo Promedio
\end{tabular}




\begin{tabular}{llll}
\hline S & Suficiencia Patrimonial & RCC & Rendimiento de Cartera \\
RB & Rentabilidad & CCCV & Cartera de Crédito Comercial por Vencer \\
EM & Eficiencia Microeconómica & VR & Variables Lingüísticas \\
GO & Gastos de Operación & TD & Términos Difusos \\
F & Fondos Disponibles & RT & Rentabilidad \\
PT & Patrimonio Promedio & ECA & Estructura y Calidad de Activos \\
\hline
\end{tabular}

Fuente: Autores

Posterior a la creación de las variables de incidencia y grados de pertenencia se establece un rango de determinación con niveles para los términos difusos que situé los valores obtenidos en tres diferentes niveles; deficientes, estables y optimo, generados a partir de los resultados obtenidos de la conversión en términos difusos de los ratios financieros del segmento 1

Tabla 6 Determinación de los Términos Difusos

\begin{tabular}{ll}
\hline & Niveles de eficiencia en Términos Difusos \\
\hline$\nearrow$ & Nivel bajo de rendimiento del ratio financiero \\
$\leftrightarrow \quad$ Nivel estable de rendimiento del ratio financiero \\
$\nwarrow \quad$ Nivel óptimo de rendimiento del ratio financiero \\
\hline
\end{tabular}

Fuente: Autores

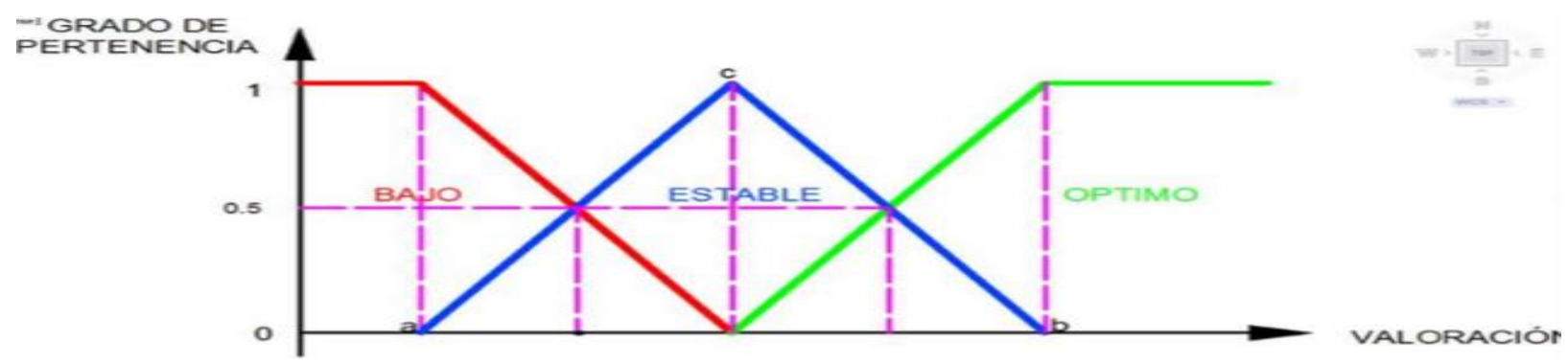

Figura 1 Niveles de eficiencia de Términos Difusos

Fuente: AutoCAD 2012

Elaborado por: Autores

Para determinar los grados de pertenecía necesitamos utilizar las funciones de pertenencia, GAMMA, L y TRIANGULAR para lo cual se presentan las siguientes expresiones algebraicas. 


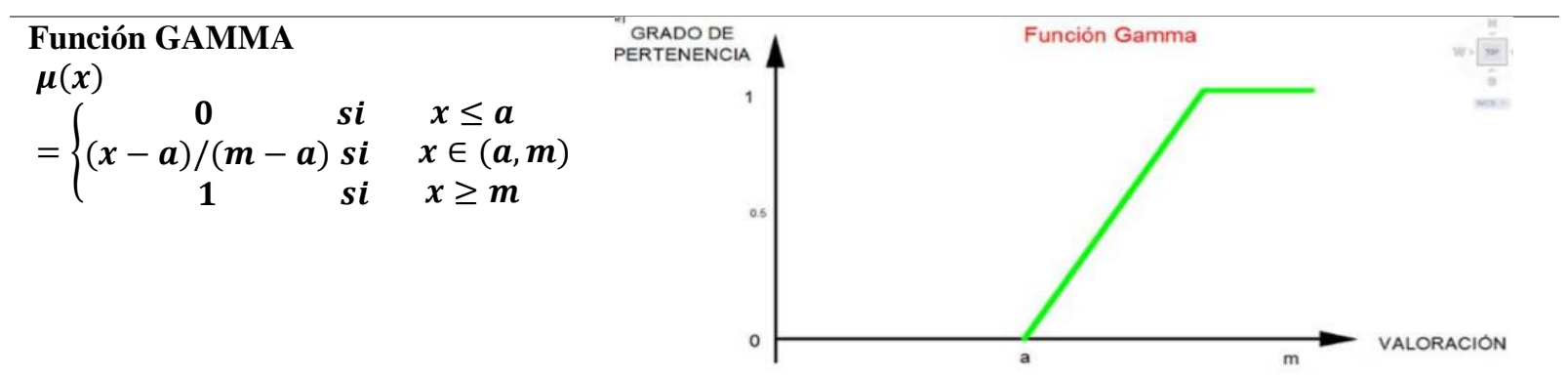

Fuente: AutoCAD 2012

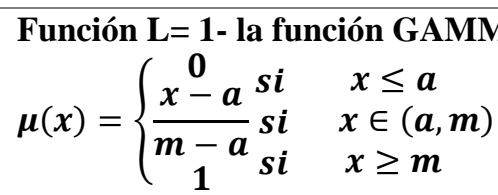

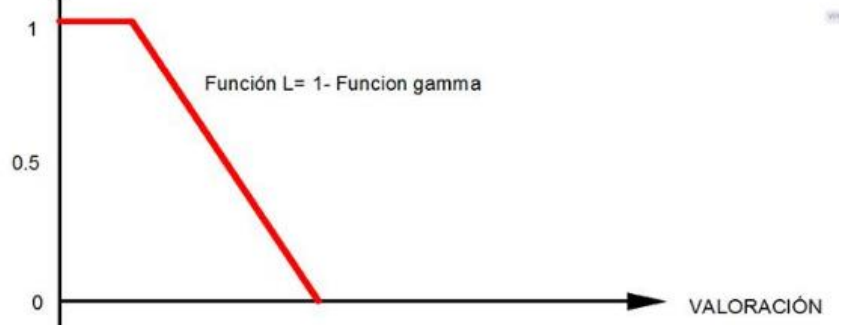

Fuente: AutoCAD 2012

$$
\begin{aligned}
& \text { Función Triangular } \\
& \boldsymbol{\mu}(\boldsymbol{x}) \\
& =\left\{\begin{array}{ccc}
0 & \text { si } & x \leq a \\
(x-a) /(m-a) & \text { si } & x \in(a, m) \\
(b-x) /(b-m) s i & x \in(m, b) \\
0 & \text { si } & x \geq b
\end{array}\right.
\end{aligned}
$$

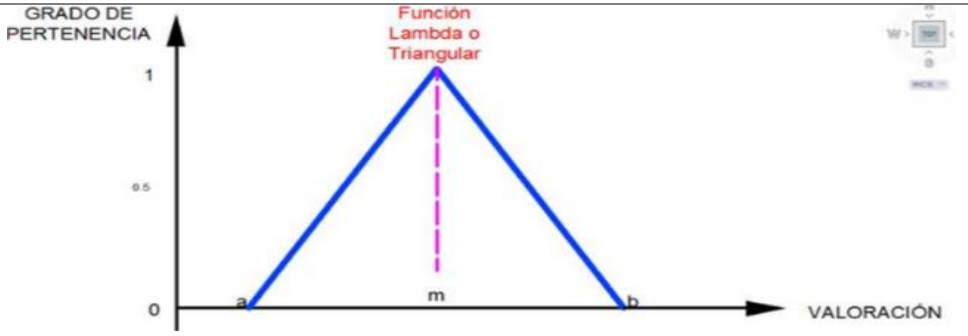

Fuente: AutoCAD 2012

Figura 2 funciones de pertenencias con su grafico de tendencia

Elaborado por: Autores a partir de (Cerra , Orizaola, \& Jimenez, 2014)

Tabla 7 Formula para establecer los rangos de la función triangular

$$
m=\frac{y_{2}-y_{1}}{x_{2}-x_{1}}
$$

Fuente: (Cerra, Orizaola, \& Jimenez, 2014).

La fórmula presentada anteriormente permite al investigador determinar los rangos que se establecerán en los grados de pertenencia "bajo", "estable" y "optimo" donde $\boldsymbol{m}$ es la pendiente de la recta de cada una de las funciones "GAMMA, L y TRIANGULAR". Una vez aplicada la formula, los rangos en los que se encuentran los ratios financieros acorde a los grados de libertas se establecieron en la Tabla 8. 
Tabla 8 Rangos para la transformación de los indicadores a términos difusos

\begin{tabular}{|c|c|c|c|c|c|c|c|c|c|c|c|c|c|}
\hline $\begin{array}{l}\text { Grado } \\
\text { de } \\
\text { Inciden } \\
\text { cia }\end{array}$ & Niveles & $\mathrm{H}$ & & & & PT & & & & $\overline{\mathrm{CC}}$ & & & \\
\hline 0 & \multirow{5}{*}{ 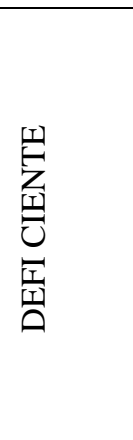 } & $\begin{array}{l}\text { Meno } \\
\mathrm{r} \mathrm{a}\end{array}$ & $\begin{array}{l}12, \\
4\end{array}$ & $\begin{array}{l}\text { Meno } \\
\text { r a }\end{array}$ & $\begin{array}{l}- \\
419,2 \\
6\end{array}$ & $\begin{array}{l}\text { Meno } \\
\mathrm{r} \mathrm{a}\end{array}$ & $\begin{array}{l}0,0 \\
5\end{array}$ & $\begin{array}{l}\text { Meno } \\
\mathrm{ra}\end{array}$ & $\begin{array}{l}- \\
11 \\
7\end{array}$ & $\begin{array}{l}\text { Meno } \\
\text { r a }\end{array}$ & $\begin{array}{l}5,7 \\
5\end{array}$ & $\begin{array}{l}\text { Meno } \\
\mathrm{ra}\end{array}$ & $\begin{array}{l}76, \\
38\end{array}$ \\
\hline 0,1 & & 12,4 & $\begin{array}{l}20, \\
4\end{array}$ & - & $\begin{array}{l}- \\
159,3\end{array}$ & 0,1 & 0,2 & $-11,7$ & $-8,7$ & 5,8 & $\begin{array}{l}11, \\
6\end{array}$ & 76,4 & $\begin{array}{l}81 \\
4\end{array}$ \\
\hline 0,2 & & 20,4 & $\begin{array}{l}28, \\
4\end{array}$ & - & 100,7 & 0,2 & 0,4 & $-8,7$ & $-5,7$ & 11,6 & $\begin{array}{l}17, \\
4\end{array}$ & 81,4 & $\begin{array}{l}86 \\
4\end{array}$ \\
\hline 0,3 & & 28,4 & $\begin{array}{l}36, \\
4\end{array}$ & 100,8 & 360,7 & 0,4 & 0,6 & $-5,7$ & $-2,7$ & 17,4 & $\begin{array}{l}23, \\
2\end{array}$ & 86,4 & $\begin{array}{l}91, \\
4\end{array}$ \\
\hline 0,399 & & 36,4 & $\begin{array}{l}44, \\
4\end{array}$ & 360,8 & 620,7 & 0,6 & 0,7 & $-2,7$ & 0,3 & 23,2 & 29 & 91,4 & $\begin{array}{l}96, \\
4\end{array}$ \\
\hline $\begin{array}{l}0,4 \\
0,699\end{array}$ & \multirow[t]{5}{*}{$\begin{array}{l}\text { ESTAB } \\
\text { LE }\end{array}$} & 44,4 & $\begin{array}{l}52, \\
4\end{array}$ & 620,8 & 880,7 & 0,7 & 0,9 & 0,3 & 3,3 & 29 & $\begin{array}{l}34, \\
9\end{array}$ & 96,4 & $\begin{array}{l}101 \\
, 4\end{array}$ \\
\hline 0,7 & & 52,4 & $\begin{array}{l}60, \\
4\end{array}$ & 880,8 & $\begin{array}{l}1140 \\
7\end{array}$ & 0,9 & 1,1 & 3,3 & 6,3 & 34,9 & $\begin{array}{l}40, \\
7\end{array}$ & 101,4 & $\begin{array}{l}106 \\
, 4\end{array}$ \\
\hline $\mathbf{0 , 8}$ & & 60,4 & $\begin{array}{l}68, \\
4\end{array}$ & $\begin{array}{l}1140, \\
8\end{array}$ & $\begin{array}{l}1400 \\
7\end{array}$ & 1,1 & 1,2 & 6,3 & 9,3 & 40,7 & $\begin{array}{l}46, \\
5\end{array}$ & 106,4 & $\begin{array}{l}111 \\
4\end{array}$ \\
\hline 0,9 & & 68,4 & $\begin{array}{l}76, \\
4\end{array}$ & $\begin{array}{l}1400, \\
8\end{array}$ & $\begin{array}{l}1660 \\
7\end{array}$ & 1,3 & 1,4 & 9,3 & $\begin{array}{l}12, \\
3\end{array}$ & 46,5 & $\begin{array}{l}52, \\
3\end{array}$ & 111,4 & $\begin{array}{l}116 \\
, 4\end{array}$ \\
\hline 1 & & 76,4 & $\begin{array}{l}84, \\
4\end{array}$ & $\begin{array}{l}1660, \\
8\end{array}$ & $\begin{array}{l}1920, \\
7\end{array}$ & 1,4 & 1,6 & 12,3 & $\begin{array}{l}15, \\
3\end{array}$ & 52,3 & $\begin{array}{l}58, \\
1\end{array}$ & 116,4 & $\begin{array}{l}121 \\
, 4\end{array}$ \\
\hline
\end{tabular}

Fuente: Autores

Una vez encontrados los intervalos y grados de pertenencia podemos establecer la relación que existen en la lógica difusa y la teoría de los conjuntos borrosos implicados en las funciones de pertenencia para el análisis de este segmento de entidades financieras y establecer ciertos criterios de aceptación o rechazo al mejoramiento de estos índices.

\section{Resultados}

De acuerdo a la metodología expuesta, se tomó los datos de los índices del total del segmento 1 de las cooperativas de ahorro y crédito del año 2015. Estos ratios fueron seleccionados en base a la Metodología CAMEL, la transformación de estos términos a términos difusos estará dada por una gráfica elaborada a partir de las formulas de la función de pertenencia y de la función triangular de cada ratio.

Tabla 9 Indicadores Financieros - Sector Financiero Popular y Solidario en (\%).

\begin{tabular}{|c|c|c|c|c|c|c|c|}
\hline Indicadores & & $\mathrm{LZ}$ & $\mathrm{S}$ & RT & ECA & RCC & EM \\
\hline Cooperativas & & $\mathrm{F}$ & $\mathrm{P}$ & PT & $\mathrm{AP}$ & $\mathrm{CCCV}$ & $\mathrm{GO}$ \\
\hline SEGMENTO 1 & 2015 & 20.8 & 351 & 0,5 & 1,2 & 11,1 & 82,1 \\
\hline
\end{tabular}

Fuente: Autores 
Mediante la función triangular de la lógica difusa se analizó cada uno de los indicadores para conocer el nivel en que se encuentran según su grado de pertenencia y establecer un nivel de mejoramiento, estos resultados tendrán un esquema elaborado en el software AUTOCAD.

\section{Liquidez}

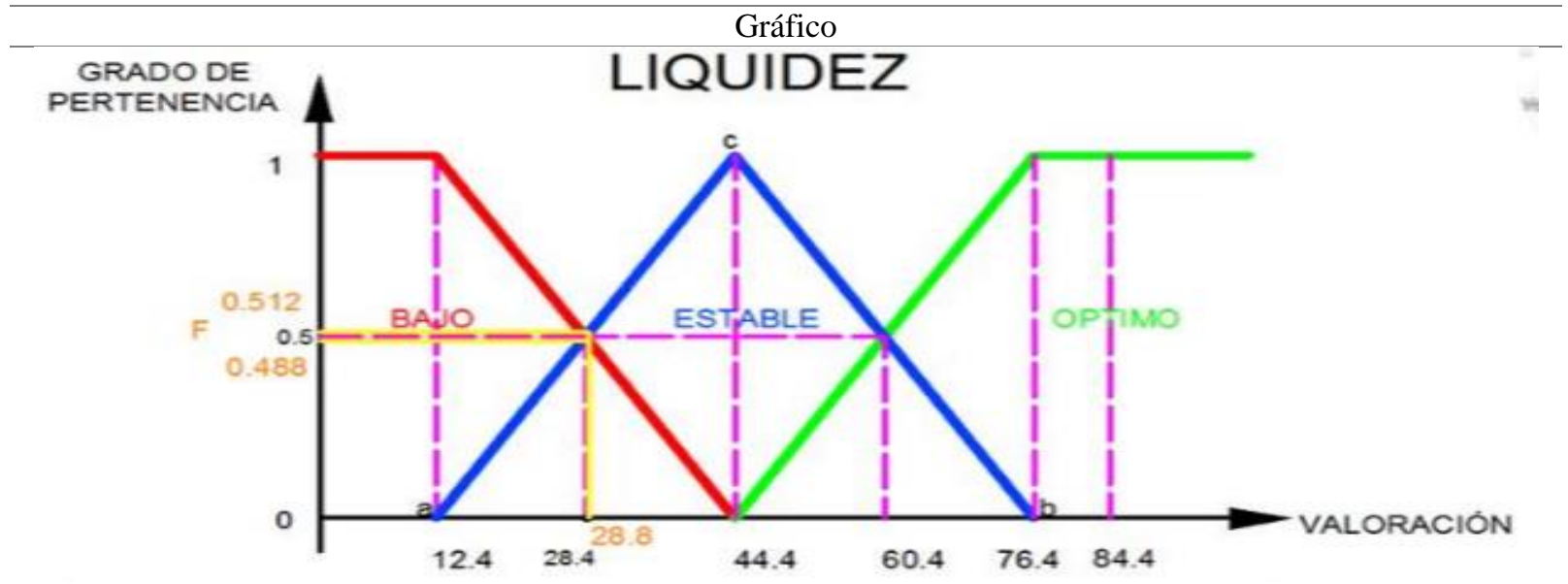

Rangos de la función triangular

$$
m=\frac{y_{2}-y_{1}}{x_{2}-x_{1}}=>\frac{1}{32}=\frac{0,5-0}{x 2-12,4}
$$$$
\text { Bajo : }(0 ; 28.4)
$$$$
\text { Estable: }(28.4 ; 60.4)
$$

Optimo: $(60.4 ; 84.4)$

Figura 3 Conversión a términos difusos de Fondos Disponibles

Fuente: AutoCAD 2012

Elaborado por: Autores

La figura 3 presenta la gráfica de Liquidez, donde se muestra el efectivo del que disponen inmediatamente las entidades de ahorro y crédito, situado un valor de 20,8\% representa un rango de 0,52 según el grado de pertenencia de la lógica difusa, lo que significa que el segmento 1 de las cooperativas posee según su variable lingüística un nivel "bajo" para el ratio de Fondos Disponibles, lo que podría representar que las entidades financieras del segmento se encuentran cerca del riesgo de no poder cumplir sus obligaciones financieras.

\section{Eficiencia Financiera}

\section{Gráfico}




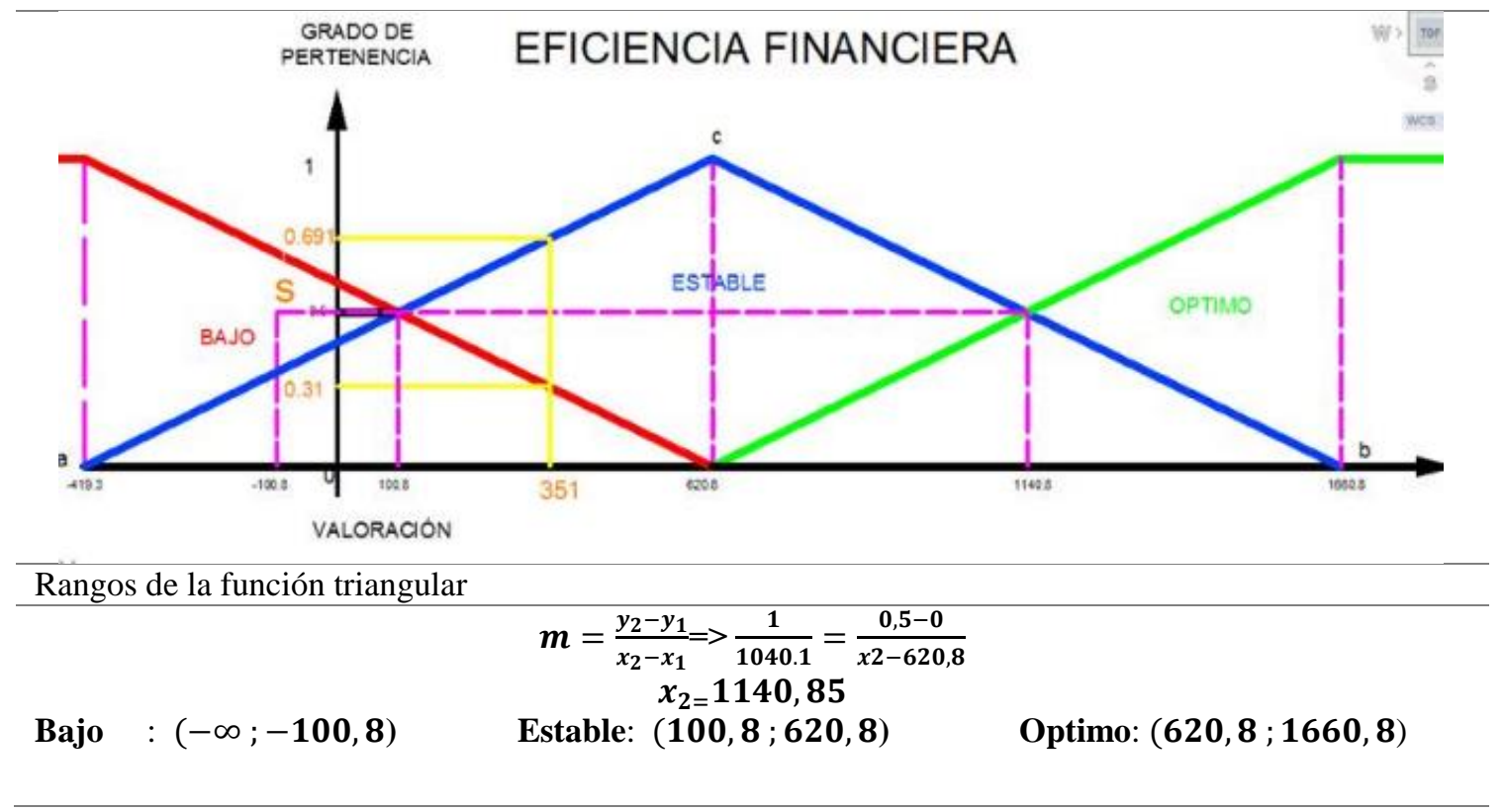

Figura 4 Conversión a términos difusos de Suficiencia Patrimonial

Fuente: AutoCAD 2012

Elaborado por: Autores

A través del grafico de conversión a términos difusos se puede determinar con respecto al ratio de eficiencia financiera el mismo que mide los ingresos financieros de una entidad con respecto a los costes; alcanzó un nivel estable con un grado de pertenencia de 0,69 es decir un valor porcentual de 351, que según la variable lingüística ubica el grado de pertenencia en un nivel estable lo que podría significar que las entidades del segmento uno son capaces de generar ganancias para cumplir con el pago de utilidades y reservas conservando de esta manera la estabilidad financiera.

\section{Rentabilidad}

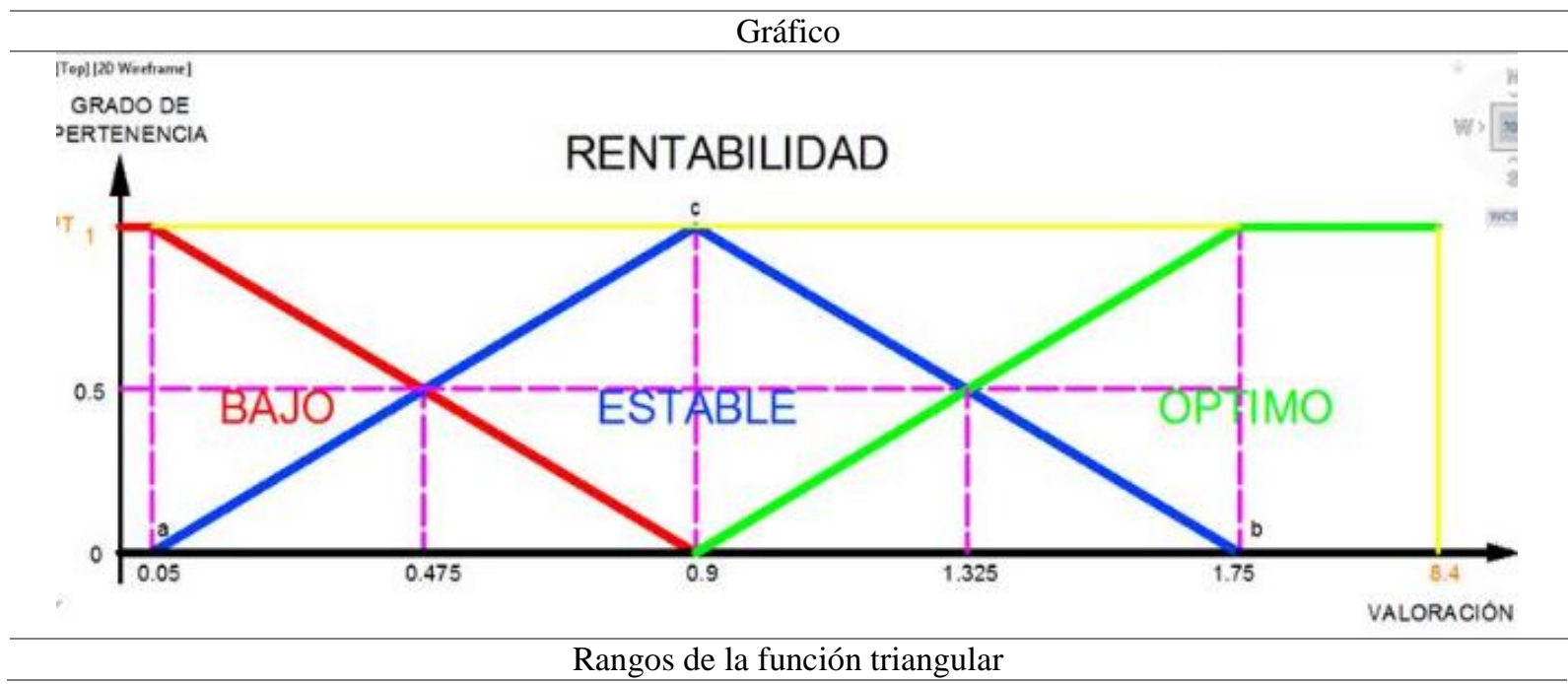




$$
m=\frac{y_{2}-y_{1}}{x_{2}-x_{1}}=>\frac{1}{0.85}=\frac{0,5-0}{x 2-0,05}
$$$$
\text { Bajo : }(0 ; 0,05)
$$

Estable: $(0,05 ; 2,175)$
Optimo: $(2,175 ; \infty)$

Figura 5 Conversión a términos difusos de Patrimonio Promedio

Fuente: AutoCAD 2012

Elaborado por: Autores

Con los resultados obtenidos del índice de rentabilidad mediante la gráfica elaborada con base en las funciones triangulares, se estableció que para el patrimonio promedio cuyo valor del segmento 8,4\% es decir un grado de pertenencia de 1 que según la variable lingüística le otorga un nivel óptimo lo que puede simbolizar que las Cooperativas del segmento tienen capacidad para generar resultados, mantener niveles de crecimiento y generar reservas.

\section{Estructura y Calidad de Activos}

Gráfico

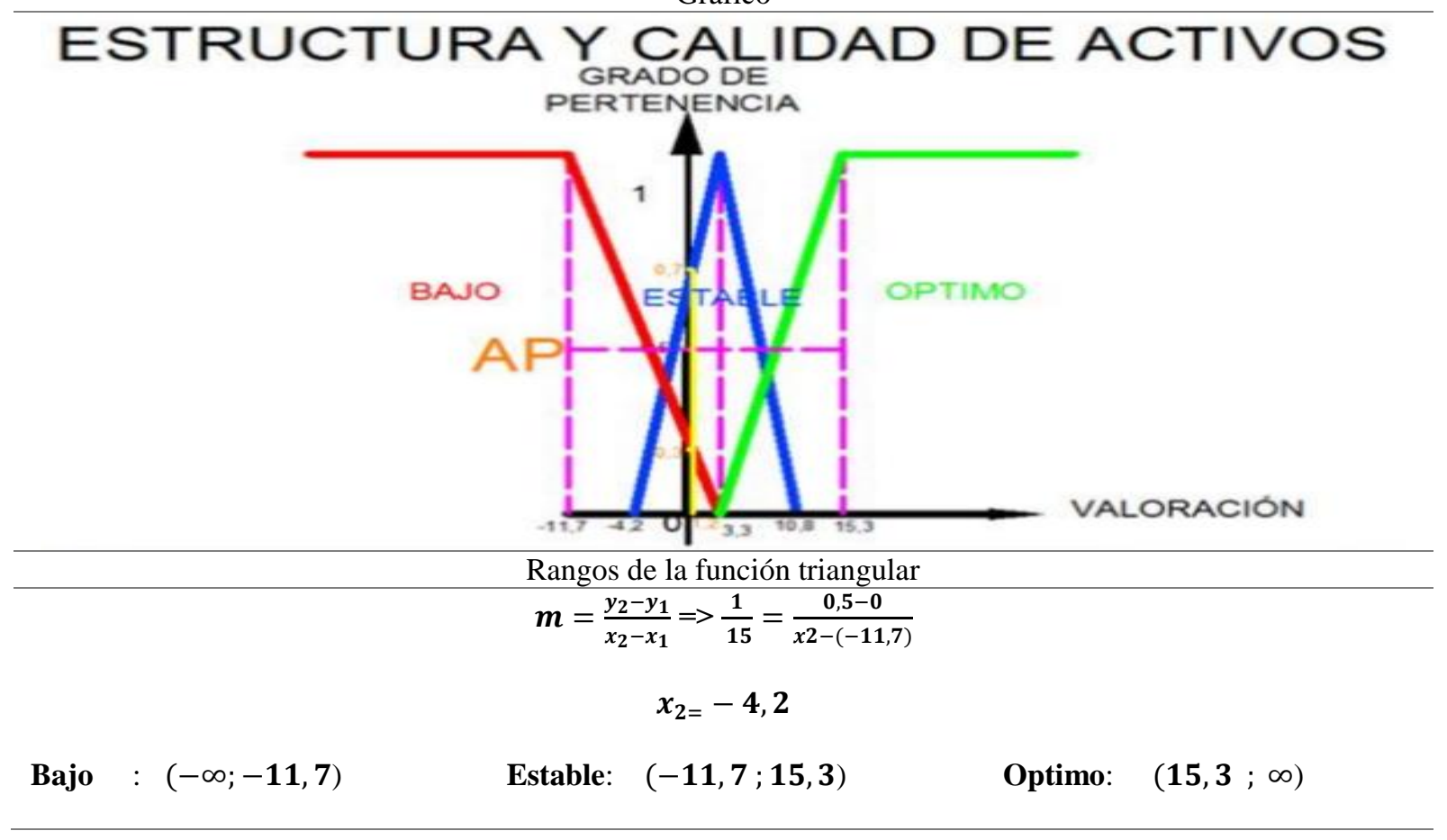

Figura 6 Conversión a términos difusos Activo Promedio

Fuente: AutoCAD 2012

Elaborado por: Autores

Con los resultados adquiridos de la gráfica de estructura y calidad de activos que proyecta los índices alcanzados al activo promedio que mide la eficiencia de una entidad en el usos de sus activos con el objeto de generar ingresos; alcanzo un porcentaje de 1,2 que en su grado de pertenencia significa 0,7 es decir que la variable lingüística bajo la que se encuentra es estable; lo 
que podría significar capacidad para generar ingresos a partir de sus activos medianamente buena.

Cobertura de Provisiones para Cartera Improductiva

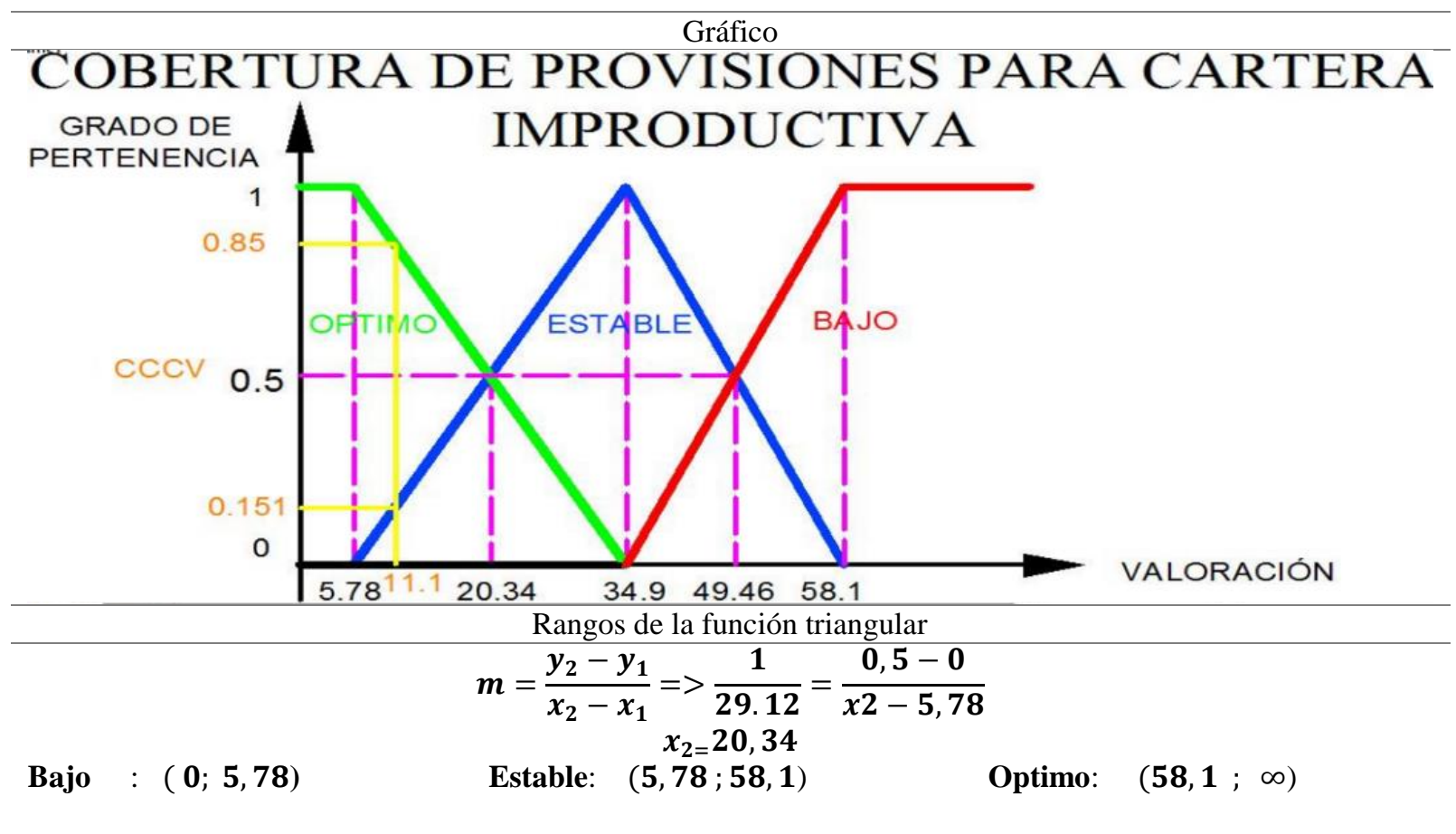

Figura 7 Conversión a términos difusos de Cartera de Crédito Comercial

Fuente: AutoCAD 2012

Elaborado por: Autores

La figura 7 muestra el grafico de conversión a términos difusos del índice de cobertura de provisiones para cartera improductiva el cual muestra a cartera de crédito comercial por vencer con un grado de pertenencia de 0,85 con respecto a los altos y con un valor porcentual de 11,1 que con base en las variables lingüísticas se ubicaría en un nivel óptimo que para cartera significaría un valor aceptable debido a que este indicador debe mantener niveles bajos dentro de las instituciones financieras lo que podría simbolizar capacidad para generar resultados operacionales que puedan cubrir gastos y generar utilidades.

\section{Eficiencia Microeconómica}




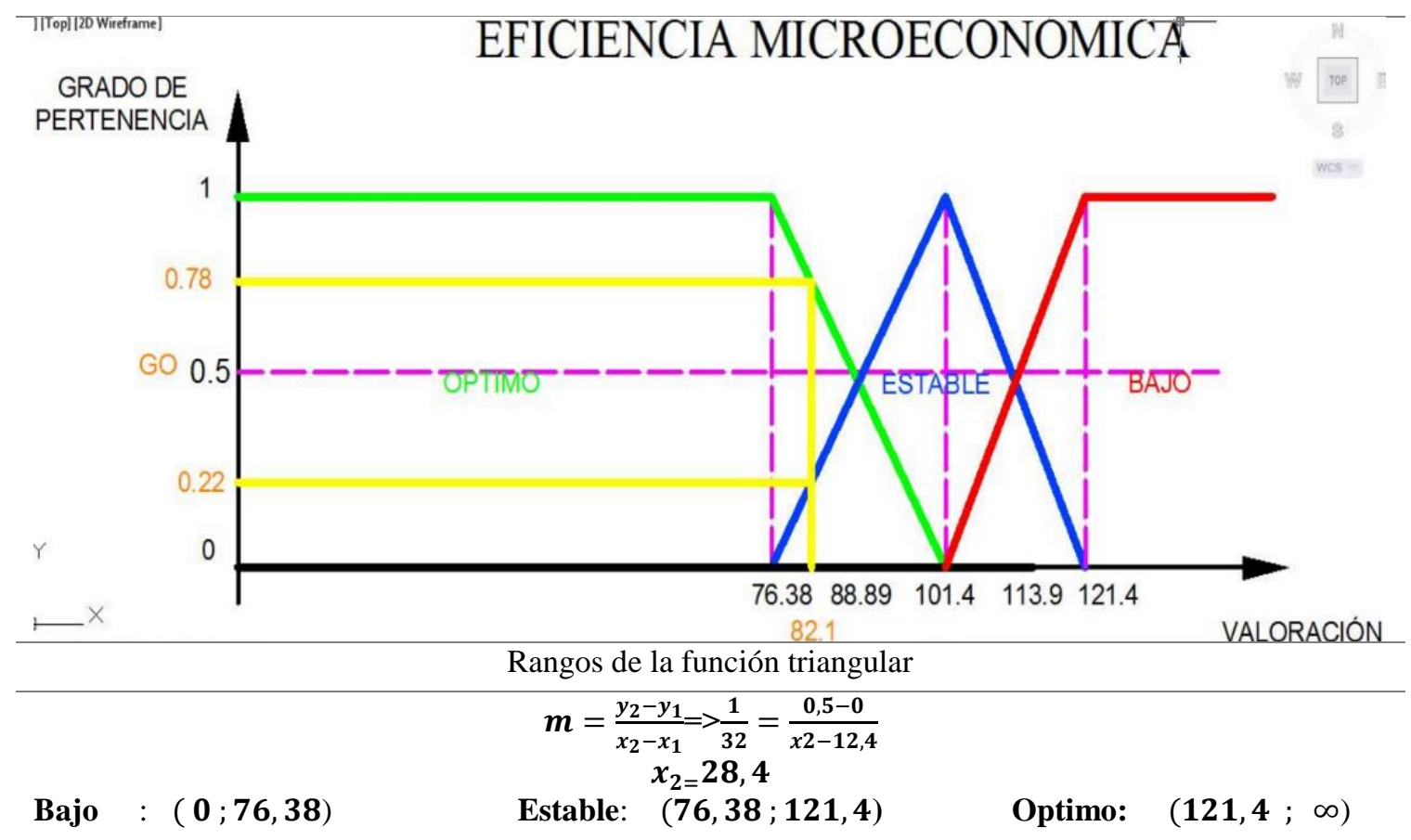

Figura 8 Conversión a términos difusos de Gastos Operacionales

Fuente: AutoCAD 2012

Elaborado por: Autores

El grafico anterior muestra la conversión a términos difusos del ratio de eficiencia microeconómica donde se puede observar que gastos operacionales se situó en un valor de $82.10 \%$ cuyo grado pertenencia se calculó en 0,78 con respecto a los óptimos que según la variable lingüística significaría un nivel excelente, lo que podría representar que el segmento tiene la capacidad de mantener rentabilidad manejando sus gastos de operación de forma adecuada.

\section{Conclusiones}

La utilización de la lógica difusa se puede convertir en un excelente medidor de rendimiento de ratios financieros, ya que puede establecer el nivel en que se encuentra cada uno, ayudando a sus evaluadores a tomar decisiones más acertadas. Una vez que se conoce el nivel de los ratios se puede establecerse políticas o medidas orientadas a la mejora de estos indicadores con el fin de brindar estabilidad a las entidades.

Al examinar la Lógica borrosa se puede decir que este postulado beneficia el análisis financiero de las cooperativas de ahorro y crédito. Ya que permite medir el desempeño de las entidades del sector con base en las metas a cumplir según el método CAMEL el cual se estableció como un nivel estándar. Este modelo difuso se utilizó para determinar el nivel económico de cada ratio en tres niveles "bajo", "estable", "optimo" dando como resultado para el ratio de liquidez un nivel bajo lo que podría significar que las entidades financieras del segmento se encuentran cerca del riesgo de no poder cumplir obligaciones financieras. Por otra parte, los ratios de eficiencia financiera y estructura y calidad de activos se encuentran en un nivel estable 
lo que podría representar que las entidades poseen capacidad sólida para cumplir con el pago de sus utilidades y generar ingresos. En cuanto al nivel óptimo en este se ubicaron los índices de rentabilidad, cobertura de provisiones para cartera improductiva y eficiencia microeconómica lo que podría denotar que las entidades del sector poseen capacidad generadora de resultados, manejando de forma adecuada sus gastos y manteniendo rentabilidad. De esta forma se puede determinar si los indicadores se encuentran en un estado saludable.

Al aplicar el modelo difuso en la información financiera en el sector cooperativo se demuestra que los ratios a nivel de cooperativas en el segmento 1 en su mayoría se encuentran entre los niveles estables y óptimos, lo que podría significar que las COAC manejan de forma adecuada sus recursos orientados al crecimiento de sus entidades dentro del sector dentro de los parámetro establecidos por la SEPS.

\section{Bibliografía}

Aceves, A. (2001). Usos y abusos de la Lógica Difusa para el control de procesos: Una alternatica para modelar lo incompleto de la informacion y lo impreciso de una observacion. Con mantenimiento productivo, 12-17.

Aching, C. (2006). Ratios Financieros y Matemáticas de la Mercadotecnia. Guia Rapida, 13-96.

Aching, C. (2012). Matemáticas financieras para la toma de decisiones. Martínez Coll, Juan.

Alarcón, A., \& Ulloa, E. (2012). Análisis de los Estados Financieros: papel en la toma de decisiones gerenciales. Observatorio de la Economía Latinoamericana(167). Obtenido de http://www.eumed.net/cursecon/ecolat/cu/2012/aaup.html

Andrade, M. (Noviembre de 2014). Análisis comparativo del sistema bancario de Ecuador con los sistemas bancarios de Colombia y Perú del periodo 2008 al 2012 utilizando el método CAMEL. Quito, Pichincha, Ecuador. Obtenido de http://repositorio.puce.edu.ec/bitstream/handle/22000/6922/7.36.000985.pdf?sequence=4

Benito Matías, T., \& Duran Vicente, M. (2010). Lógica Borrosa. Madrid: Universidad Carlos III. Obtenido de http://www.it.uc3m.es/jvillena/irc/practicas/08-09/10.pdf

Bernal, D., \& Amat, O. (2012). Anuario de ratios financieros sectoriales en México para analisis comparativo empresarial. $R a \quad$ Ximhai, 271-286. Obtenido de http://www.redalyc.org/pdf/461/46125172003.pdf

Blass, J. (17 de 02 de 1993). La interferencia linguistica en Valencia. Valencia: Dkategoria. Obtenido de http://delta.cs.cinvestav.mx/ gmorales/ldifll/node2.html

Buniak, L. (s.f.). Un nuevo enfoque para el Analisis y la Calificacion del Riego Bancario en el Contexto de Basilea II. Obtenido de CAMELSR: http://www.camelsr.com/Portals/0/pdf/specials/Nuevo-Enfoque-para-Analizar-Calificarel-Riesgo-Bancario-CAMELSBCOR.pdf 
Cerra , J., Orizaola, P., \& Jimenez, M. (2014). Utilización de la lógica borrosa en la selección de personas e ideas para la participacion en programas publicos de ayuda a la creación de empresas. Cuadernos de Gestión, 77-98.

D`Negri, C. A., \& De Vito, E. L. (2006). Introducción al razonamiento aproximado: lógica difusa. Revista Americana de Medicina Respiratoria, 6(3), 126-136.

Duarte, O. (2000). Aplicaciones de la Lógica Difusa. Dialnet, 5-12. Obtenido de https://dialnet.unirioja.es/descarga/articulo/4902776.pdf

Galindo, J. (2002). Conjuntos y sistemas difusos. Departamento de lenguajes y Ciencias de la computación universidad de Malaga, 1-5. Obtenido de http://www.lcc.uma.es/ ppgg/FSS/FSS5.pdf

Garcia, J., Trinidad, J., \& Gomez, J. (05 de 03 de 2004). El método de las dos funciones de distrbución: la version trapezoidal. Obtenido de http://www.ual.es/personal/jetrini/objetos/trapezoidal.pdf

Gonzales, C. (18 de marzo de 2012). Lógica Difusa. Obtenido de http://www.esi.uclm.es/www/cglez/downloads/docencia/2011_Softcomputing/LogicaDif usa.pdf

Guzman, D., \& Castaño, V. (2006). La lógica difusa en ingenieria: principios, aplicaciones y futuro. Ciencia y tecnologia, 88-107.

Haank, S. (1996). Fuzzy logic. Chicago: ISBN.

Hurtado, L., Quintero, O., \& Garcia , J. (2014). Fuzzy Logic. revista de metodos cuantitativos para la economia y la emprea, 54-87.

Jiménez, L., Vallejo, D., Albusac, J., Castro-Schez, J. J., \& Glez-Morcillo, C. (2011). A multiagent architecture for supporting distributed normality-based intelligent surveillance. Engineering Applications of Artificial Intelligence, 24(2), 325-340. doi:dx.doi.org/10.1016/j.engappai.2010.11.005

Kaufmann, A., \& Gil-Aluja, J. (1988). Modelos para la investigacion de efectos olvidados. Candariña: Milladoiro.

La Hora. (30 de Abril de 2006). Cooperativas, una necesidad. La Hora.

LOEPS. (2011). Asamblea Nacional Constituyente. Quito: Registro Oficial N. 444.

Lopez , D. (20 de 11 de 2013). CIBERTEC. Obtenido de https://my.laureate.net/faculty/webinars/Documents/Ingenieria2013/November2013_Logi ca\%20Difusa.pdf

Marzan, H. (2011). La Función Gamma. Intec, 1-69. 
Miño Grijalva, W. (2013). Historia del cooperativismo en el Ecuador. Quito: Editogran S.A. Obtenido de http://www.politicaeconomica.gob.ec/wpcontent/uploads/downloads/2013/04/Libro-Cooperativismo-Final-op2-Altaresolucio\%CC\%81n.pdf

Morales, G. (2002). Introducción a la Lógica Borrosa. Centro de investigacion y estudios avanzados del IPN, 1-12. Obtenido de http://casanchi.com/mat/intrologicadifusa01.pdf

Mullor, J. (2000). Lógica borrosa y su a plicacion a la contaboilidad. Revista española de financiación y contabilidad, 83-106.

Nava, R., \& Marbelis, A. (2009). Análisis Financiero: una herramienta clave para una gestion financiera eficiente. Revista Venezolana de Gerencia, 606-628. Obtenido de http://www.scielo.org.ve/pdf/rvg/v14n48/art05.pdf

Pérez, I., \& León, B. (2007). Lógica difusa para pricipiantes. Caracas: Texto C.A. Obtenido de https://books.google.com.ec/books?id=iCiiyyCFZuIC\&pg=PA16\&dq=funciones+de+per tenencia+logica+difusa\&hl=es\&sa=X\&ved=0ahUKEwjy-

dyIy7bOAhXC6iYKHVfnB04Q6AEIHDAA\#v=onepage \&q=funciones\%20de\%20perten encia\%20logica\%20difusa\&f=false

PNFPEES, P. N., SENAMI, S. N., \& PNUD, P. d. (2012). Finanzas populares y solidarias impulsa el desarollo. Economia y Finanzas Populares y Solidarias para el Buen Vivir, 6-15.

Revista Lideres. (28 de junio de 2015). La economía popular y solidaria gana participación. Revista Lideres. Obtenido de http://www.revistalideres.ec/lideres/economia-popular-cooperativagana-participacion.html

Reyes Vera, R. (1994). Evolución de las Finanzas y la Actividad Financiera de México. Mexico: Laguna. Obtenido de file:///C:/Users/USUARIO/Downloads/capitulo3.pdf

Richardson, D. (2009). Sistema de monitoreo PERLAS. Consejo Mundial de Cooperativas de Ahorro y Credito Serie de Herramientas tecnicas(4), 1-32. Obtenido de http://webcache.googleusercontent.com/search?q=cache:lpHbuZcUw6oJ:www.woccu.or g/functions/view_document.php\%3Fid\%3DPEARLS_Monograph$\mathrm{SP}+\& \mathrm{~cd}=1 \& \mathrm{hl}=\mathrm{es} \& \mathrm{ct}=\mathrm{clnk} \& \mathrm{gl}=\mathrm{ec}$

Santandreu, E. (2000). El análisis de la empresa a través de los ratios. 4-18. Obtenido de http://www.centrem.cat/ecomu/upfiles/publicacions/analisi.pdf

SEPS. (2015). Super Intendencia de Economia Popular y Solidaria. Quito. Obtenido de Resolución No 038-2015-F

Trillas, E., \& Gutierrez , J. (1992). Aplicación de la lógica borrosa. Madrid: Raycar S.A.

Trillas, E., \& Gutierrez, J. (1992). Aplicaciones de la logica borrosa. Madrid: C.S.I.C. Obtenido de https://books.google.com.ec/books?hl=es\&lr\&id=Uvhx3hMh5wMC\&oi=fnd\&pg=PR8\& 
$\mathrm{dq}=\operatorname{logica} \% 20$ difusa $\% 201$ ibros\&ots $=21 \mathrm{u}-$

h8G4ac\&sig=TgTyT3siRUDiEpmQoqEiwG9MTeA\#v=onepage\&q\&f=false

Unidad de Gestión del Riesgo del Sistema Financiero. (Septiembre de 2015). Banco Central del Ecuador. Recuperado el 18 de Julio de 2016, de www.bce.ec: https://contenido.bce.fin.ec/documentos/PublicacionesNotas/Catalogo/Cuadernos/Cuad13 5.pdf

Villegas, E. (2002). Análisis Financiero en los Agronegocios. Revista Mexicana de Agronegocios, 337-346. 